\title{
Dynamical instability of fluid spheres in the presence of a cosmological constant
}

\author{
C. G. Böhmerı \\ The Erwin Schrödinger International Institute for Mathematical Physics, \\ Boltzmanngasse 9, A-1090 Wien, Austria and \\ Institut für Theoretische Physik, Technische Universität Wien, \\ Wiedner Hauptstr. 8-10, A-1040 Wien, Austria \\ T. Harkd ${ }^{\dagger}$ \\ Department of Physics, The University of Hong Kong, \\ Pokfulam Road, Hong Kong SAR, P. R. China
}

(Dated: March 10, 2019)

\begin{abstract}
The equations describing the adiabatic, small radial oscillations of general relativistic stars are generalized to include the effects of a cosmological constant. The generalized eigenvalue equation for the normal modes is used to study the changes in the stability of the homogeneous sphere induced by the presence of the cosmological constant. The variation of the critical adiabatic index as a function of the central pressure is studied numerically for different trial functions. The presence of a large cosmological constant significantly increases the value of the critical adiabatic index. The dynamical stability condition of the homogeneous star in the Schwarzschild-de Sitter geometry is obtained and several bounds on the maximum allowable value for a cosmological constant are derived from stability considerations.
\end{abstract}

PACS numbers: $97.30 . \mathrm{Sf}, 97.10 . \mathrm{Cv}, 97.10 . \mathrm{Sj}$

*Electronic address: boehmer@hep.itp.tuwien.ac.at

†Electronic address: harko@hkucc.hku.hk 


\section{INTRODUCTION}

The possibility that the cosmological constant be nonzero and dominates the energy density of the universe today is one of the most intriguing problems of the contemporary physics. Considered already in 1896 by von Seeliger and Neumann (see Schunck and Mielke [1] and references therein), who added it to the Poisson equation for the Newtonian potential to compensate the energy density of the 'aether', introduced by Einstein [2] in his equations of general relativity to obtain a static model of the Universe, eliminated by him after the discovery of the redshift of the stars by Hubble, the cosmological constant was reintroduced next by Bondi and Gold [3] and Hoyle and Narlikar [4] to resolve an age crisis and to construct a universe that satisfied the "Perfect Cosmological Principle". The cosmological constant appeared again in inflationary models of the universe introduced by Guth [5]. The density of the positive isotropic energy density of the scalar field (inflaton) that dominates the first stages of the evolution of the Universe behaves like a cosmological constant leading to a rapid cosmological expansion of the universe during a de Sitter phase [6] .

Within the classical general relativity, the existence of a cosmological constant is equivalent to the postulate that the total energy momentum tensor of the Universe $T_{i k}^{(U)}$ possesses an additional piece $T_{i k}^{(V)}$, besides that of its matter content $T_{i k}^{(m)}$, of the form $T_{i k}^{(V)}=\Lambda g_{i k}$, where generally one may assume that the cosmological constant $\Lambda$ is a scalar function of space and time coordinates. Such a form of the additional piece $T_{i k}^{(V)}$ has previously been obtained in certain field-theoretical models and is interpreted as a vacuum contribution to the energy momentum tensor [7, 8, 9, 10],

$$
\Lambda g_{i k}=\frac{8 \pi G}{c^{4}}\left\langle T_{i k}^{(V)}\right\rangle=\frac{8 \pi G}{c^{4}}\left\langle\rho_{V}\right\rangle g_{i k}
$$

where $\rho_{V}$ is the energy of the vacuum (see Padmanabhan [11] for a recent review of the cosmological constant problem). The vacuum value of $T_{i k}$ thus appears in the form of a cosmological constant $\Lambda$ in the gravitational field equations. In the framework of standard general relativity the contracted Bianchi identity requires $\Lambda$ to be a constant. However, generalized physical models with time and space varying cosmological constant (decaying vacuum energy) have been intensively investigated in the physical literature [12].

A natural thermal interpretation of the cosmological constant has been proposed based on the fact that the de Sitter vacuum can be thought as being hot, since even a geodesic observer in this manifold will detect an isotropic background of thermal radiation with 
temperature $T_{V}=\left(\Lambda / 12 \pi^{2}\right)^{1 / 2}[13]$. The cosmological constant can thus be interpreted as a parameter measuring the intrinsic temperature of the empty space-time, or in a sense, of the geometry itself.

The first pressing piece of data, which motivated a reconsideration of the cosmological constant involved the study of Type Ia Supernovae. Observations of Type Ia Supernovae with redshift up to about $z \sim 1$ provided evidence that we may live in a low mass-density Universe, with the contribution of the non-relativistic matter (baryonic plus dark) to the total energy density of the Universe of the order of $\Omega_{m} \sim 0.3$ [14, 15, 16]. The value of $\Omega_{m}$ is significantly less than unity [17], and consequently either the Universe is open or there is some additional energy density $\rho$ sufficient to reach the value $\Omega_{\text {total }}=1$, predicted by inflationary theory. Observations also show that the deceleration parameter of the Universe $q$ is in the range $-1 \leq q<0$, and the present-day Universe undergoes an accelerated expansionary evolution [18].

Several physical models have been proposed to give a consistent physical interpretation to these observational facts. One candidate, and maybe the most convincing one for the missing energy is the vacuum energy density or the cosmological constant $\Lambda$ [2, 11]. The presence of a cosmological constant implies that, for the first time after inflation, in the present epoch its role in the dynamics of the Universe becomes dominant [19].

On the other hand there is the possibility that scalar fields present in the early universe could condense to form the so named boson stars. There are also suggestions that the dark matter could be made up of bosonic particles. This bosonic matter would condense through some sort of Jeans instability to form compact gravitating objects. A boson star can have a mass comparable to that of a neutron star [20]. A detailed analysis of the mass dependence of boson stars on the self-interacting potential done by Mielke and Schunck [21] has shown that the mass $M$ of the bosons stars can range from $M=10^{10} \mathrm{~kg}$ for mini-boson stars with radius $R \approx 10^{-18} \mathrm{~m}$ to values that can reach or extend the limiting mass of $3.23 M_{\odot}$ of the neutron stars. For the very light universal axion of the effective string models, the gravitational mass is in the range of $\sim 0.5 M_{\odot}$. The density of a mini-boson star can exceeds by a factor of $10^{45}$ the density of a neutron star.

In general, a boson star is a compact, completely regular configuration with structured layers due to the anisotropy of scalar matter, an exponentially decreasing 'halo', a critical mass inverse proportional to the constituent mass, an effective radius and a large particle 
number (for a detailed review of the boson star properties see [22]). The simplest kind of boson star is made up of a self-interacting complex scalar field $\Phi$ describing a state of zero temperature. The self-consistent coupling of the scalar field to its own gravitational field is via the Lagrangian [22],[23], [24],

$$
L=\frac{1}{2 k} \sqrt{-g} R+\frac{1}{2} \sqrt{-g}\left[g^{i k} \Phi_{; i}^{*} \Phi_{; k}-V\left(|\Phi|^{2}\right)\right],
$$

where $V\left(|\Phi|^{2}\right)$ is the self-interaction potential usually taken in the form $V\left(|\Phi|^{2}\right)=$ $m^{2}|\Phi|^{2}+\lambda|\Phi|^{4} / 4$. Here $m$ is the mass of the scalar field particle (the boson) and $\lambda$ is the self-interaction parameter. For the bosonic field the stationarity ansatz is assumed, $\Phi(t, r)=\phi(r) \exp (-i \omega t)$. If we suppose that in the star's interior regions and for some field configurations $\Phi$ is a slowly varying function of $r$, so that it is nearly a constant, then in the gravitational field equations the scalar field will play the role of a cosmological constant, which could also describe a mixture of ordinary matter and bosonic particles.

At interplanetary distances, the effect of the cosmological constant could be imperceptible. However, Cardona and Tejeiro [25] have shown that a bound of $\Lambda$ can be obtained using the values observed from the Mercury's perihelion shift in a Schwarzschild-de Sitter space-time, which gives $\Lambda<10^{-55} \mathrm{~cm}^{-2}$. For compact general relativistic objects the cosmological constant modifies the mass-radius ratio $M / R$ of compact general relativistic objects. Generalized Buchdahl type inequalities for $M / R$ in the presence of a cosmological constant have been derived by Mak et al. [26] and by Böhmer [27], see also Stuchlík [28]. Thus, for a non-zero $\Lambda$, the mass-radius ratio is given by $2 M / R \leq 4 / 9+(2 / 3)\left(4 / 9-\Lambda R^{2} / 3\right)^{1 / 2}$ [27]. For a recent discussion of the astrophysical bounds on the cosmological constant obtained from the study of spherically symmetric bodies see Balaguera-Antolínez et al. [30].

In a very influential paper, Chandrasekhar [31] studied the infinitesimal adiabatic oscillations of a gaseous sphere in a general relativistic framework. In particular, from stability considerations he derived a condition on the radius $R$ of the stable dense general relativistic star, which must obey the constraint $R>\left(2 G M / c^{2}\right) K /(\gamma-4 / 3)$, where $\gamma$ is the ratio of the specific heats of the matter and $K$ is a constant which depends on the equation of state of the matter. For homogeneous spheres $K=19 / 42$, while for polytropic stars $K$ has values in the range $K=0.565(n=1)$ and $K=1.124(n=3)$. The stability against small radial oscillations of equilibrium configurations of cold, gravitationally bound states of complex scalar fields with equilibrium configurations exhibiting a mass profile against central den- 
sity similar to that of ordinary neutron stars was studied by Gleiser and Watkins [32]. By studying the behavior of the eigenfrequencies of the perturbations for different values of the central density $\sigma$ it was shown by using both analytical and numerical methods that configurations with central densities greater than a given value $\sigma_{c}(0)$ are unstable against radial perturbations. The stability of boson star solutions in a $D$-dimensional, asymptotically anti-de Sitter space-time in the presence of a cosmological term was discussed by Astefanesei and Radu [33]. It was found that for $D>3$ the boson star properties are close to those in four dimensions with a vanishing cosmological constant. A different behavior was noticed for the solutions in the three dimensional case.

It is the purpose of the present paper to study the effects of the possible existence of a cosmological constant on the radial oscillations of a dense general relativistic star. As a first step we generalize the pulsation equation to include $\Lambda$. This equation is used to study the effect of $\Lambda$ on homogeneous compact astrophysical objects. The values of the critical adiabatic index $\gamma$ are estimated numerically for different values of the cosmological constant $\Lambda$. The stability criterion of general relativity for a homogeneous fluid sphere is generalized to take into account the presence of the cosmological constant. Based on the analysis of the solutions of the gravitational field equations for a constant density sphere some other simple criteria of stability for compact objects in the presence of a cosmological constant are obtained.

This paper is organized as follows. In Section 2, using the Einstein gravitational field equations, we derive the eigenvalue equation for adiabatic radial pulsations for the case $\Lambda \neq 0$. In Section 3 we discuss the mass continuity and Tolman-Oppenheimer-Volkoff (TOV) equations in the presence of a cosmological constant and the solution for the homogeneous sphere is obtained. The stability of the homogeneous star is considered in Section 4 . The results are discussed and summarized in Section 5.

\section{ADIABATIC RADIAL PULSATIONS OF RELATIVISTIC STARS IN THE SCHWARZSCHILD-DE SITTER GEOMETRY}

In the present Section we will develop the general formalism for the study of perturbations of the exact solutions of Einstein's field equations for fluid spheres in the presence of a cosmological constant, by following the approach introduced by Chandrasekhar [31]. We 
shall consider only perturbations that preserve spherical symmetry. Under these perturbations only radial motions will ensue. The metric of the corresponding space-time can be taken in the form

$$
d s^{2}=e^{\nu}\left(d x^{0}\right)^{2}-e^{\lambda} d r^{2}-r^{2}\left(d \theta^{2}+\sin ^{2} \theta d \phi^{2}\right)
$$

where $\nu$ and $\lambda$ are functions of $x^{0}=c t$ and of the radial coordinate $r$ only.

The energy-momentum tensor for a spherically symmetric space-time is

$$
T_{i}^{k}=(\epsilon+p) u_{i} u^{k}-p \delta_{i}^{k}
$$

where $\epsilon$ is the energy density of the matter and $p$ is the thermodynamic pressure. $u^{i}=d x^{i} / d s$ is the four-velocity.

The gravitational field equations corresponding to the line element given by Eq. (3) and with the cosmological constant included [34], together with the 0th order equations, corresponding to the static equilibrium case, are presented in Appendix A .

Writing $\lambda=\lambda_{0}+\delta \lambda, \nu=\nu_{0}+\delta \nu, \rho=\rho_{0}+\delta \rho$ and $p=p_{0}+\delta p$, where the index 0 refers to the unperturbed metric and physical quantities, we find that to the first order the perturbed components of the energy-momentum tensor are given by $T_{0}^{0}=\epsilon, T_{i}^{i}=-p, i=1,2,3$ (no summation $), T_{0}^{1}=\left(\epsilon_{0}+p_{0}\right) v$ and $T_{1}^{0}=\left(\epsilon_{0}+p_{0}\right) v e^{\lambda_{0}-\nu_{0}}$, where $v=d r / d x^{0}$.

By introducing the "Lagrangian displacement" $\xi$ defined as $v=\partial \xi / \partial t$, we obtain the variations of the metric functions and of the energy density in a form similar to the $\Lambda=0$ case: $\delta \lambda=-\left(\lambda_{0}^{\prime}+\nu_{0}^{\prime}\right) \xi,(\delta \nu)^{\prime}=\left[\delta p /\left(\epsilon_{0}+p_{0}\right)-\left(\nu_{0}^{\prime}+1 / r\right) \xi\right]\left(\lambda_{0}+\nu_{0}\right)$ and $\delta \epsilon=-\left(1 / r^{2}\right)\left[r^{2}\left(\epsilon_{0}+p_{0}\right) \xi\right]^{\prime}$, respectively.

In order to obtain an expression for $\delta p$ we need to impose an extra condition on the system. This condition is the law of conservation of the baryon number density $n$, which can be written as $\left(n u^{i}\right)_{; i}=0$. Generally $n$ is a function of both energy density and pressure, $n=n(\epsilon, p)$. By taking $n=n_{0}(r)+\delta n\left(x^{0}, r\right)$ it follows that the variation of the pressure can be obtained as

$$
\delta p=-\xi \frac{d p_{0}}{d r}-\gamma p_{0}\left[\frac{\exp \left(\nu_{0} / 2\right)}{r^{2}}\right]\left[r^{2} \exp \left(-\nu_{0} / 2\right) \xi\right]^{\prime},
$$

where $\gamma$ is the adiabatic index defined as [31]

$$
\gamma=\left(p \frac{\partial n}{\partial p}\right)^{-1}\left[n-(\epsilon+p) \frac{\partial n}{\partial \epsilon}\right]
$$


With the use of the previous results for the perturbed quantities, and by assuming that all the perturbations have a dependence on $x^{0}$ of the form $\exp \left(i \omega x^{0}\right)$, from the linearized Einstein field equations we obtain the Sturm-Liouville eigenvalue equation for the eigenmodes in the presence of a cosmological constant as

$$
\frac{d}{d r}\left(\Pi \frac{d \zeta_{j}}{d r}\right)+\left(Q+\omega_{j}^{2} W\right) \zeta_{j}=0, j=1,2, \ldots, n,
$$

where we have redefined the "Lagrangian displacement" $\xi$ as $\xi \rightarrow r^{-2} \exp [\nu / 2(r)] \zeta($ corresponding to a Lagrangian displacement of the radial coordinate of the form $\delta r\left(x^{0}, r\right)=$ $r^{-2} \exp [\nu / 2(r)] \zeta \exp \left(i \omega x^{0}\right)$ and we have denoted

$$
\begin{gathered}
\Pi=\frac{\gamma p}{r^{2}} e^{[\lambda(r)+3 \nu(r)] / 2}, \\
Q=\frac{\Pi}{\gamma p}\left[\frac{p^{2}}{\epsilon+p}-\frac{4 p^{\prime}}{r}-\left(\frac{8 \pi G}{c^{4}} p-\Lambda\right)(\epsilon+p) e^{\lambda(r)}\right] \\
W=\frac{\epsilon+p}{r^{2}} e^{[3 \lambda(r)+\nu(r)] / 2} .
\end{gathered}
$$

The boundary conditions for $\zeta(r)$ are that $\zeta(r) / r^{3}$ is finite or zero as $r \rightarrow 0$ and that the Lagrangian variation of the pressure $\Delta p=-\left(\gamma p e^{\nu(r) / 2} / r^{2}\right) d \zeta / d r$ vanishes at the surface of the star.

For all stellar models of physical interest the frequency spectrum of the normal radial modes is discrete; the $n$-th normal mode has $n$ nodes between the center and the surface of the star. The normal mode eigenfunctions are orthogonal with respect to the weight function $W(r): \int_{0}^{R} W \zeta_{j} \zeta_{k} d r=0$, if $j \neq k$.

In the presence of a cosmological constant the system of equations governing infinitesimal adiabatic radial pulsations is given by

$$
\begin{aligned}
& \frac{d \xi}{d r}=-\frac{1}{r}\left(3 \xi+\frac{\Delta p}{\gamma p}\right)-\frac{\xi}{\epsilon+p} \frac{d p}{d r}, \\
& \frac{d \Delta p}{d r}=\left[\frac{\omega^{2}}{c^{2}} e^{\lambda(r)-\nu(r)}(\epsilon+p) r-4 \frac{d p}{d r}\right] \xi+ \\
& {\left[\frac{r}{\epsilon+p}\left(\frac{d p}{d r}\right)^{2}-e^{\lambda(r)}\left(\frac{8 \pi G}{c^{4}} p-\Lambda\right)(\epsilon+p) r\right] \xi+} \\
& {\left[\frac{1}{\epsilon+p} \frac{d p}{d r}-\frac{4 \pi G}{c^{4}}(\epsilon+p) r e^{\lambda(r)}\right] \Delta p . }
\end{aligned}
$$


In Eqs. (11) and (12) $\xi$ is the relative radial displacement $\xi=\Delta r / r$, where $\Delta r$ is the radial displacement. $\Delta p$ is the corresponding radial displacement of the pressure. Eqs. (11) and (12) represents the generalization to the case of the Schwarzschild-de Sitter geometry of the oscillations equations introduced by Gondek et al. [35].

These pulsation equations have to be integrated with the boundary conditions $(\Delta p)_{\text {center }}=$ $-(3 \gamma p \xi)_{\text {center }}$ and $(\Delta p)_{\text {surface }}=0$.

\section{STATIC FLUID SPHERES WITH NON-ZERO COSMOLOGICAL CON- STANT}

The basic equations describing the equilibrium of a static fluid sphere in the presence of a cosmological constant are given by Eqs. (A5)-(A7) in Appendix A. These equations have to be considered together with the equation $\nu_{0}^{\prime}=-2 p_{0}^{\prime} /\left(\epsilon_{0}+p_{0}\right)$, which can be obtained from the condition $T_{i ; k}^{k}=0$, describing the conservation of the energy momentum tensor.

With the use of the gravitational field equations and of the conservation equation the Tolman-Oppenheimer-Volkoff (TOV) equation, describing the hydrostatic equilibrium of a fluid sphere in the presence of a cosmological constant $\Lambda \neq 0$, can be immediately obtained in the form [26, 29, 36]

$$
p_{0}^{\prime}=-\frac{\left(\epsilon_{0}+p_{0}\right)\left[\frac{G}{c^{2}} M(r)+\left(\frac{4 \pi G}{c^{4}} p_{0}-\frac{\Lambda}{3}\right) r^{3}\right]}{r^{2}\left[1-\frac{2 G M(r)}{c^{2} r}-\frac{\Lambda}{3} r^{2}\right]},
$$

where we have denoted

$$
M(r)=\frac{4 \pi}{c^{2}} \int_{0}^{r} \epsilon_{0} r^{2} d r .
$$

For the TOV equation for boson stars see Schunck and Mielke [22].

To obtain a dimensionless form of Eq. (13) and of the mass continuity equation, $d M / d r=$ $\left(4 \pi / c^{2}\right) \epsilon_{0} r^{2}$, we introduce a dimensionless independent variable $\eta$ and the dimensionless functions $\varepsilon_{0}(\eta), P_{0}(\eta)$ and $m(\eta)$ by means of the transformations $r=a \eta, \epsilon_{0}=\epsilon_{c} \varepsilon_{0}(\eta)$, $p_{0}=\epsilon_{c} P_{0}(\eta)$ and $M=M^{*} m(\eta)$, respectively. Here $a$ is a scale factor (a characteristic length), $\epsilon_{c}$ the central energy density of the star and $M^{*}$ a characteristic mass. With the use

of these transformations we obtain the mass continuity and TOV equations in the following dimensionless form

$$
\frac{d P_{0}(\eta)}{d \eta}=-\frac{\left[\varepsilon_{0}(\eta)+P_{0}(\eta)\right]\left[m(\eta)+\left(P_{0}(\eta)-l\right) \eta^{3}\right]}{\eta^{2}\left[1-\frac{2 m(\eta)}{\eta}-l \eta^{2}\right]},
$$




$$
\frac{d m}{d \eta}=\eta^{2} \varepsilon_{0}(\eta)
$$

where we denoted

$$
a^{2}=\frac{c^{4}}{4 \pi G \epsilon_{c}}, M^{*}=\frac{4 \pi \epsilon_{c} a^{3}}{c^{2}}, l=\frac{\Lambda c^{4}}{12 \pi G \epsilon_{c}} .
$$

Eqs. (13) and (14) must be integrated with the boundary conditions $p_{0}(R)=0$ and $M(0)=0$, respectively, where $R$ is the radius of the fluid sphere, together with an equation of state of the form $p_{0}=p_{0}\left(\epsilon_{0}\right)$. For a wide class of equations of state one can prove uniqueness and existence of these solutions, see [29, 37].

In the new variables the equation of state becomes $P_{0}=P_{0}\left(\varepsilon_{0}\right)$, while the boundary conditions are given by $m(0)=0$ and $P_{0}\left(\eta_{S}\right)=0$, where $\eta_{S}=R / a$ is the value of the dimensionless radial coordinate $\eta$ at the surface of the star. If we suppose that $m$ is an increasing function of $\eta$, while $\varepsilon_{0}$ and $P_{0}$ are decreasing functions of the same argument, then it follows that generally $\varepsilon_{0} \in[1,0]$ and $P_{0} \in\left[P_{c}, 0\right]$, where $P_{c}=P_{0}(0)=p_{0}(0) / \epsilon_{c}$ is the value of the pressure at the center of the star.

A particularly important fluid sphere configuration is the one corresponding to the constant density case, $\epsilon=\epsilon_{c}=$ constant. This condition gives $\varepsilon_{0}=1, \forall \eta \in\left[0, \eta_{S}\right]$, and the mass continuity equation can be integrated immediately to give

$$
m(\eta)=\frac{\eta^{3}}{3}
$$

The solution of the TOV equation with the boundary condition $P_{0}(0)=P_{c}$ is given by

$$
P_{0}(\eta)=\frac{(1 / 3-l) \alpha-\sqrt{1-(2 / 3+l) \eta^{2}}}{\sqrt{1-(2 / 3+l) \eta^{2}}-\alpha},
$$

where we denoted

$$
\alpha=\frac{1+P_{c}}{1 / 3-l+P_{c}} .
$$

The radius $R$ of the star can be obtained from the condition $P_{0}\left(\eta_{S}\right)=0$ and is given by

$$
R=a \frac{\sqrt{6 P_{c}\left(2 P_{c}+1\right)-9 P_{c}\left(P_{c}+2\right) l}}{3 P_{c}+1-3 l} .
$$

The values of the central pressure $P_{c}$ depend on the physically allowed upper limit for the pressure. If we consider the classical restriction of general relativity, $p \leq \epsilon / 3$, as $p \geq 0$, it follows that $P_{c} \in[0,1 / 3]$. A more general restriction can be obtained by assuming that $p \leq \epsilon$, the upper limit being the strong equation of state for the hot nucleonic gas. It is believed 
that matter actually behaves in this manner at densities above about ten times the nuclear density, that is, at densities greater than $10^{17} \mathrm{~g} / \mathrm{cm}^{3}$ and at temperatures $T=(\epsilon / \sigma)^{1 / 4}>10^{13}$ $\mathrm{K}$, where $\sigma$ is the radiation constant [7]. In this case $P_{c} \in[0,1]$. Hence generally $P_{c} \leq w$, where $w \in[0,1]$. Consequently, $\alpha \in[3 /(1-3 l), 6 /(4-3 l)]$.

The metric coefficient $\exp \left(-\lambda_{0}\right)$ is given by

$$
\exp \left[-\lambda_{0}(\eta)\right]=1-(2 / 3+l) \eta^{2} .
$$

From the Bianchi identity and the matching across the vacuum boundary of the star it follows that $\exp [\nu(\eta)]=\left[1-(2 / 3+l) \eta_{S}^{2}\right] /\left[1+P_{0}(\eta)\right]^{2}$, or, equivalently,

$$
e^{\nu_{0}(\eta)}=\frac{(1 / 3-l)^{2}}{(2 / 3+l)^{2}}\left[e^{-\frac{\lambda_{0}(\eta)}{2}}-\alpha\right]^{2} \text {. }
$$

\section{STABILITY OF HOMOGENEOUS STARS IN THE PRESENCE OF A COS- MOLOGICAL CONSTANT}

The eigenvalue problem formulated in Eq. (7) can be re-expressed in a well-known variational form: the extremal values of the quantity

$$
\omega^{2}=\frac{\int_{0}^{R}\left[\Pi\left(\frac{d \zeta}{d r}\right)^{2}-Q \zeta^{2}\right] d r}{\int_{0}^{R} W \zeta^{2} d r}
$$

are the eigenvalues $\omega_{j}^{2}$ of Eq. (7) and the functions $\zeta(r)$ which give the extremal values are the corresponding eigenfunctions. A sufficient condition for the dynamical instability of a mass is that the right hand side of Eq. (24) vanishes for some chosen trial function $\zeta$ which satisfies the boundary conditions [31], [38].

In terms of the system of variables introduced in the previous Section Eq. (24) takes the form

$$
\begin{gathered}
a^{2} \omega^{2} \int_{0}^{\eta_{S}} \frac{\left(1+P_{0}\right)}{\eta^{2}} e^{\frac{3 \lambda+\nu}{2}} \zeta^{2} d \eta=\int_{0}^{\eta_{S}} \gamma P_{0} \frac{e^{\frac{\lambda+3 \nu}{2}}}{\eta^{2}} \zeta^{\prime 2} d \eta- \\
\int_{0}^{\eta_{S}} \frac{e^{\frac{\lambda+3 \nu}{2}}}{\eta^{2}}\left[\frac{P_{0}^{\prime 2}}{1+P_{0}}-\frac{4 P_{0}^{\prime}}{\eta}-2\left(P_{0}-\frac{3}{2} l\right)\left(1+P_{0}\right) e^{\lambda}\right] \zeta^{2} d \eta .
\end{gathered}
$$

Setting $\omega^{2}=0$ and assuming that the adiabatic index is constant throughout the star, we obtain the value of the critical adiabatic index $\gamma_{c}$ as

$$
\gamma_{c}=\frac{\int_{0}^{\eta_{S}} \frac{e^{\frac{\lambda+3 \nu}{2}}}{\eta^{2}}\left[\frac{P_{0}^{\prime 2}}{1+P_{0}}-\frac{4 P_{0}^{\prime}}{\eta}-2\left(P_{0}-\frac{3}{2} l\right)\left(1+P_{0}\right) e^{\lambda}\right] \zeta^{2} d \eta}{\int_{0}^{\eta_{S}} P_{0} \frac{e^{\frac{\lambda+3 \nu}{2}}}{\eta^{2}} \zeta^{\prime 2} d \eta} .
$$


For $\gamma<\gamma_{c}$ dynamical instability occurs and the star collapses. For a homogeneous star in the presence of a cosmological constant the equilibrium metric coefficients and the pressure distribution have been obtained in the previous Section. In order to find the range of values of $\gamma_{c}$ we need to chose some explicit functional forms for $\zeta$. For the trial function $\zeta=\eta^{3}$ the variation of $\gamma_{c}$ as a function of the central pressure $P_{c}$ is represented, for different values of l, in Fig. 1.

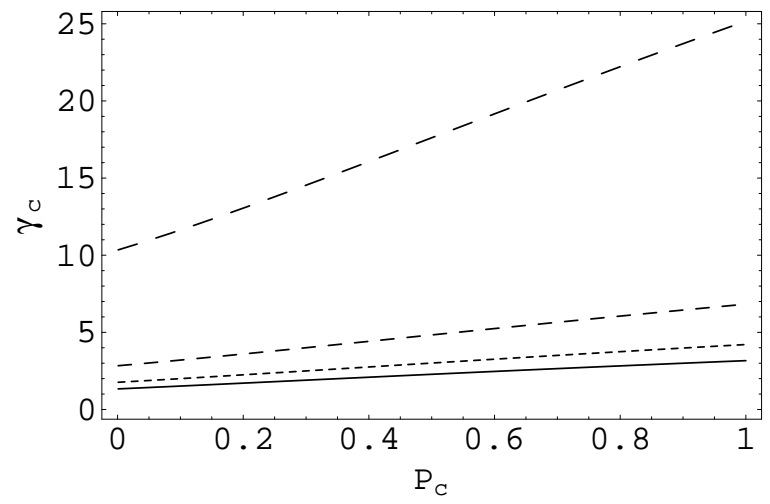

FIG. 1: Critical adiabatic index $\gamma_{c}$ as a function of the central pressure $P_{c}$ for the trial function $\zeta=\eta^{3}$ and for different values of the cosmological constant: $l=0$ (solid curve), $l=0.1$ (dotted curve), $l=0.2$ (dashed curve) and $l=0.3$ (long dashed curve).

In terms of the gravitational radius of the star $R_{g}=2 G M_{S} / c^{2}$, where $M_{S}=4 \pi \epsilon_{c} R^{3} / c^{2}$ is the total mass of the star, and of the radius $R$, the critical adiabatic index $\gamma_{c}$ can be represented as a power series in $R_{g} / R$ of the form

$$
\begin{aligned}
\gamma_{c}= & \frac{\frac{4}{3}-l}{1-3 l}+\frac{19}{42}\left(1-\frac{21}{19} l\right)\left(\frac{R_{g}}{R}\right) \\
& +\frac{146}{441}\left[1-\frac{87}{73} l\left(1+\frac{21}{58} l\right)\right]\left(\frac{R_{g}}{R}\right)^{2} \\
& +O\left[\left(\frac{R_{g}}{R}\right)^{3}\right]
\end{aligned}
$$

For the trial function $\zeta=\eta^{3} \exp (-\nu / 4)$ Fig. 2 represents $\gamma_{c}$. 


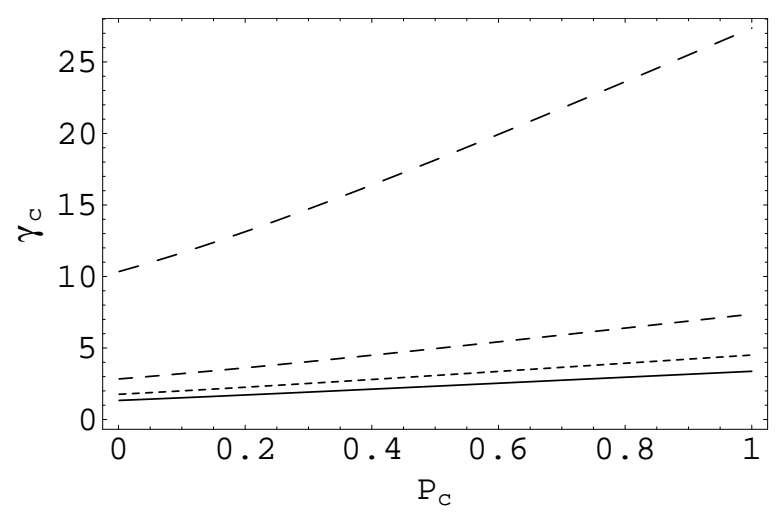

FIG. 2: Critical adiabatic index $\gamma_{c}$ as a function of the central pressure $P_{c}$ for the trial function $\zeta=\eta^{3} \exp (-\nu / 4)$ and for different values of the cosmological constant: $l=0$ (solid curve), $l=0.1$ (dotted curve), $l=0.2$ (dashed curve) and $l=0.3$ (long dashed curve).

In this case the representation of $\gamma_{c}$ in terms of $R_{g} / R$ is given by

$$
\begin{aligned}
\gamma_{c}= & \frac{\frac{4}{3}-l}{1-3 l}+\frac{19}{42}\left(1-\frac{21}{19} l\right)\left(\frac{R_{g}}{R}\right) \\
& +\frac{5401}{15876}\left[1-\frac{26691}{21604} l\left(1+\frac{447}{1271} l\right)\right]\left(\frac{R_{g}}{R}\right)^{2} \\
& +O\left[\left(\frac{R_{g}}{R}\right)^{3}\right] .
\end{aligned}
$$

In first order in $R_{g} / R$ both Eqs. (27) and (28) give the same result. This shows that the Chandrasekhar stability limit [31] is generally independent of the form of the trial function.

Lastly, $\gamma_{c}$ as a function of both $l$ and $P_{c}$ and for the trial function $\zeta=\eta^{3}$, is represented in Fig. 3.

For a homogeneous sphere and for the trial function $\zeta=\eta^{3}$ all the integrals in Eq. (25) can be calculated exactly. The corresponding pulsation equation and the details of the calculations are presented in Appendix B.

Performing a series expansion of Eq. (B1) in Appendix B, keeping only the terms up to the second order in $\theta_{S}$ and neglecting the terms containing $l^{2}$ we obtain the equation

$$
\gamma-\frac{4}{3}-(3 \gamma-1) l=\frac{390 \gamma-406-3(142+303 \gamma) l}{378\left(\frac{2}{3}+l\right)} \theta_{S}^{2}
$$

Taking $l=0$ gives

$$
\gamma-\frac{4}{3}=\frac{390 \gamma-406}{378} \eta_{S}^{2}
$$




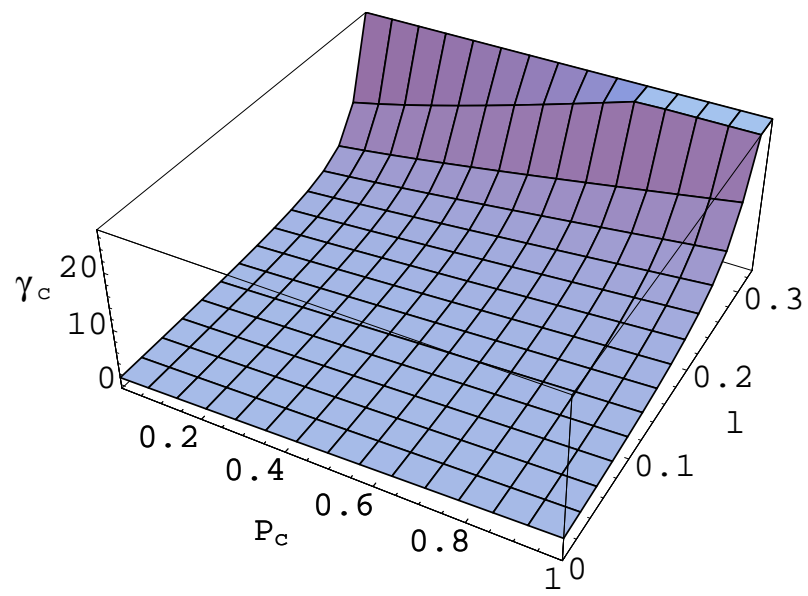

FIG. 3: Critical adiabatic index $\gamma_{c}$ as a function of the central pressure $P_{c}$ and of $l$ for the trial function $\zeta=\eta^{3}$

Therefore

$$
\gamma-\frac{4}{3}<\frac{19}{42} \times \frac{2 G M_{S}}{c^{2} R}
$$

or, equivalently,

$$
R<\frac{19}{42(\gamma-4 / 3)} R_{g}
$$

Hence from Eq. (29) we have recovered the stability condition derived by Chandrasekhar [31] for $\Lambda=0$. For $l \neq 0$ we obtain

$$
R<\frac{19}{42} \frac{1-273 l / 19}{\gamma-4(1+9 l / 4) / 3} R_{g}
$$

To obtain Eq. (33) we have approximated $\gamma$ in all terms containing the product $\gamma l$ by $\gamma=4 / 3$. Eq. (33) represents the condition for the dynamical stability of a massive general relativistic object in the presence of a cosmological constant. It generalizes to the case $\Lambda \neq 0$ the relation initially derived by Chandrasekhar [31]. In the limit $l=0$ we obtain again Eq. (32).

Alternatively, we may divide Eq. (25) by the integral on its left-hand side and proceed as described above. Up to terms of the order $\theta_{S}^{2}$ and $l$ this yields

$$
a^{2} \omega^{2}=\gamma-\frac{4}{3}-l(3 \gamma-1)-\frac{54 \gamma-53-15(4+9 \gamma) l}{63(2 / 3+l)} \theta_{S}^{2},
$$

which generalizes the original Eq. (77) of Chandrasekhar [31]. 
Approximating, as before, $\gamma$ in all terms containing $\gamma l$ by $\gamma=4 / 3$ and letting $\omega \rightarrow 0$ we arrive at

$$
R<\frac{19}{42} \frac{1-240 l / 19}{\gamma-4(1+9 l / 4) / 3} R_{g}
$$

For the trial function $\xi=\eta^{3} \exp (-\nu / 4)$ the integrals in Eq. (25) can also be calculated exactly. A series expansion in terms of $\theta_{S}$ of the result for this choice gives

$$
\gamma-\frac{4}{3}-(3 \gamma-1) l=\frac{123 \gamma-107-3(65+102 \gamma) l}{189(2 / 3+l)} \theta_{S}^{2} .
$$

For $l=0 \mathrm{Eq}$. (36) gives the same limit as obtained for the case of the trial function $\xi=\eta^{3}$, represented by Eq. (32). For $l \neq 0$, by approximating again $\gamma$ by $4 / 3$ in the terms containing the product $\gamma l$, we obtain

$$
R<\frac{19}{42} \frac{1-201 l / 19}{\gamma-4(1+9 l / 4) / 3} R_{g}
$$

which differs only slightly in the numerical value of the coefficient of $l$ from the previous result. As was already noted, the slight differences in the approximations show that firstly, the stability limits are generally independent of the trial function and that secondly, they are quite unaffected by the 'location' where the higher order terms are neglected.

By imposing the condition $\gamma<\gamma_{c}$ in Eqs. (27) and (28) gives an other form of the stability criterion for high density relativistic stars in the presence of the cosmological constant

$$
R<\frac{19}{42} \frac{1-21 l / 19}{\gamma-(4 / 3-l)(1-3 l)^{-1}} R_{g}
$$

The denominator of the latter stability criterion coincides with those derived before, if terms containing $l^{2}$ are neglected in a series expansion.

\section{DISCUSSIONS AND FINAL REMARKS}

The hypothesis that a non-zero cosmological constant could play an important role in the structure of compact stellar objects seems to be difficult to accommodate with the smallness of the present determined or postulated values of this physical quantity. While a non-vanishing cosmological constant, if definitely confirmed, would carry a significant implication for our understanding of the structure and global dynamics of the Universe as a whole, the ways in which one can clearly test it in an astrophysical or astronomical setting are very limited. Peebles [39] has shown that the cosmological constant has little effect 
on the local dynamics. The effects on the galactic dynamics of $\Lambda$ can hardly be observed also with distant clusters, because it appears to be always cancelled in observable quantities [40]. But, on the other hand, the effects of the cosmological constant could be important for stellar objects in the far past, most of the cosmological models with decaying vacuum energy predicting a time variable and decreasing cosmological constant [41]. Therefore, the interior structure of early stars could have been strongly influenced by the presence of a cosmological constant.

In the presence of a cosmological constant the radius $R$ of the homogeneous stellar configuration is related to the gravitational (Schwarzschild) radius of the star $R_{g}$ by the relation

$$
R=\frac{\left(3 P_{c}+1-3 l\right)^{2}}{P_{c}\left[2\left(2 P_{c}+1\right)-3\left(P_{c}+2\right) l\right]} R_{g} .
$$

From the restriction $P_{c} \leq 1$ on the pressure we find the following simple stability criterion for a homogeneous star in the presence of a cosmological constant

$$
R \geq \frac{(3 w+1-3 l)^{2}}{w[2(2 w+1)-3(w+2) l]} R_{g} .
$$

For $w=1 / 3$ we have $R \geq 9(2-3 l)^{2} R_{g} /(10-21 l)$, while for $w=1$ we obtain $R \geq$ $(4-3 l)^{2} R_{g} /[3(2-3 l)]$.

The condition of the non-negativity of the radius of the star, $R \geq 0$, imposes the constraint $l=\Lambda c^{4} / 12 \pi G \epsilon_{c}<2(2 w+1) / 3(w+2)$ on the cosmological constant, or, equivalently,

$$
\Lambda<\frac{(2 w+1)}{(w+2)} \frac{8 \pi G \epsilon_{c}}{c^{4}} .
$$

For a star composed from matter having a density equivalent to the typical nuclear density, $2 \times 10^{14} \mathrm{~g} / \mathrm{cm}^{3}$, Eq. (41) leads to an upper limit of the cosmological constant equal to

$$
\Lambda<3 \times 10^{-13} \mathrm{~cm}^{-2}
$$

In the presence of a cosmological constant the dynamical stability condition is modified according to Eq. (33). The critical value $\gamma_{c}^{\prime}=4 / 3$ of the adiabatic index is replaced by $\gamma_{c}^{\prime}=(4 / 3)(1+9 l / 4)=\left(1+3 \Lambda c^{4} / 16 \pi G \epsilon_{c}\right)$. A large value of $l$ can increase significantly the value of $\gamma_{c}^{\prime}$. This could be the case for mixtures of the potential energy dominated bosonic matter and usual matter, when the cosmological constant $\Lambda$ can be interpreted as the static equilibrium value of the scalar field potential, $\Lambda=V\left(\left|\Phi_{0}\right|^{2}\right)=$ const. In this case $l$ could have 
a large value, even of the order of unity. The presence of the bosonic component strongly affects the dynamic stability of the system. The critical values of $\gamma_{c}$ of configurations with given $\epsilon_{c}$ require that the radius of the star exceeds a certain lower limit $R$, if the configuration is to be stable. In the presence of a cosmological constant instability will occur when $\gamma$ is in excess of $4(1+9 l / 4) / 3$ by a small amount. Since for a perfect gas the maximum admissible value for $\gamma$ is $\gamma=5 / 3$, the stability condition imposes the restriction

$$
\left[5-4\left(1+\frac{9 l}{4}\right)\right]>0
$$

or, equivalently,

$$
\Lambda<\frac{4 \pi G \epsilon_{c}}{3 c^{4}} .
$$

By using the same restriction on $\gamma$ in Eq. (38) we obtain

$$
\Lambda<\frac{\pi G \epsilon_{c}}{c^{4}} .
$$

These conditions, obtained from stability considerations, differs from the condition given by Eq. (41) only by factors of the order of unity.

The properties of boson stars and the influence of the cosmological term on their properties in arbitrary $D$-dimensional, asymptotically anti de Sitter space-times have been recently considered in [33]. By assuming for the spherically symmetric bound state of the scalar field a stationary ansatz $\Phi=\phi(r) \exp (-i \sigma t)$ the metric for the boson star can be chosen in the form $d s^{2}=F(r) d r^{2}+r^{2} d \Omega_{D-2}^{2}-F(r) \exp [-2 \delta(r)] d t^{2}$, where $d \Omega_{D-2}^{2}=\omega_{a b} d x^{a} d x^{b}$ is the line element of a unit $(D-2)$-dimensional sphere and $F(r)=1-2 m(r) / r^{D-3}-2 \Lambda r^{2} /(D-2)(D-1)$. The function $m(r)$ is related to the local mass-energy density up to some dimensiondependent factor. With the help of these parameters one can define the particle number $N=\left\{4 \pi^{(D-1) / 2} / \Gamma[(D-1) / 2]\right\} \int_{0}^{\infty} \phi^{2} e^{\delta} F^{-1} r^{D-2} d r$ and the effective radius of the boson star as $R=\left\{4 \pi^{(D-1) / 2} / \Gamma[(D-1) / 2] N\right\} \int_{0}^{\infty} \phi^{2} e^{\delta} F^{-1} r^{D-1} d r$. For $\Lambda=0$, boson stars are characterized by an exponential decay of the scalar field, for which the mass term in the potential is responsible. For $\Lambda \neq 0$ the situation is quite different; for $r \rightarrow \infty$, one finds that $m(r) \sim M+A r^{2 C+D-1}$, where $A$ and $C$ are some constants depending on the dimensionality of the space-time $D$ and $\Lambda$, whose exact form is given in [33], and $M$ is the ADM mass of the star. Thus, the cosmological constant implies a complicated power decay at infinity rather than an exponential one as found in the asymptotically flat space case. The maximum mass $M_{\max }$ and the maximum particle number $N_{\max }$ for the boson star decreases with the value 
of the cosmological constant, but the general properties of the solution are the same as in the $\Lambda=0$ case.

The analysis of the stability of the boson stars follows the standard approach, by assuming that the scalar field and the metric tensor components can be perturbed in the usual way, with the perturbation of the scalar field written as $\delta \phi=f_{1}(t, r)+i \phi_{0}(r) \dot{g}(t, r)$. Then the field equations and the Klein-Gordon equation can be linearized to a system of two self-adjoint coupled equations, the pulsation equations for the boson stars. These equations have to be integrated with the boundary conditions $r^{2} g^{\prime} \rightarrow 0$ at the origin and $f_{1} \rightarrow 0, g^{\prime} r^{2 C+D} \rightarrow 0$ for $r \rightarrow \infty$.

By assuming that all perturbations are of the form $\exp (i \chi t)$, where $\chi$ is the characteristic frequency to be determined, one can again formulate the stability problem of the boson stars in the presence of a cosmological constant as a variational problem, with the eigenvalues $\chi^{2}$ forming a discrete sequence $\chi_{0}^{2} \leq \chi_{1}^{2} \leq \ldots \leq \chi_{n}^{2}$. The numerical results show that there is a critical value of the central density of the star $\phi_{c}(0)$ so that $\chi^{2}>0$ for central values of the scalar field smaller than $\phi_{c}(0)$ while other configurations are unstable.

Due to the close mathematical and even physical analogy between the two eigenvalue problems for boson and "normal" stars, we expect that stability conditions of the form given by Eqs. (33), (35) and (38) should also exist in the case of boson stars. For boson stars the corresponding mass appearing in the stability conditions is the ADM mass $M$, and the radius is defined as above. The coefficient $\gamma$ becomes a scalar field-dependent quantity $\gamma_{B S}$, which could be given as a function of the the central density of the star $\phi_{c}(0), \gamma_{B S}=\gamma\left[\phi_{c}(0)\right]$. The values of the numerical coefficients could be also very different as compared to normal stars. Therefore for boson stars a mass-radius relation of the form $R<\left[f(l) /\left(\gamma_{B S}-\right.\right.$ const $\left.)\right] M$ is a direct consequence of the variational formulation of the stability problem and of the boundary conditions, requiring the vanishing of some quantities at center of the star and infinity, respectively. But finding exact analytical stability conditions for boson stars requires further mathematical and numerical investigations.

\section{Acknowledgments}

The work of C. G. B. was supported by the Junior Research Fellowship of The Erwin Schrödinger International Institute for Mathematical Physics. 
The work of T. H. was supported by a Seed Funding Programme for Basic Research of the Hong Kong Government.

\section{APPENDIX A: FIELD EQUATIONS}

For a spherically symmetric matter distribution the gravitational field equations are given by

$$
\begin{gathered}
\left(r e^{-\lambda}\right)^{\prime}=1-\frac{8 \pi G}{c^{4}} T_{0}^{0} r^{2}-\Lambda r^{2}, \\
\nu^{\prime}=\frac{e^{\lambda}-1}{r}-\frac{8 \pi G}{c^{4}} T_{1}^{1} r-\Lambda r \\
-\frac{1}{2} e^{-\lambda}\left(\nu^{\prime \prime}+\frac{\nu^{\prime 2}}{2}+\frac{\nu^{\prime}-\lambda^{\prime}}{r}-\frac{\nu^{\prime} \lambda^{\prime}}{2}\right) \\
+\frac{1}{2} e^{-\nu}\left(\ddot{\lambda}+\frac{\dot{\lambda}^{2}}{2}-\frac{\dot{\lambda} \dot{\nu}}{2}\right)=\frac{8 \pi G}{c^{4}} T_{2}^{2}+\Lambda, \\
e^{-\lambda} \frac{\dot{\lambda}}{r}=\frac{8 \pi G}{c^{4}} T_{0}^{1} .
\end{gathered}
$$

The zeroth order (or static equilibrium) equations are

$$
\begin{gathered}
\left(r e^{-\lambda_{0}}\right)^{\prime}=1-\frac{8 \pi G}{c^{4}} \epsilon_{0} r^{2}-\Lambda r^{2}, \\
\nu_{0}^{\prime}=\frac{e^{\lambda_{0}}-1}{r}+e^{\lambda_{0}}\left(\frac{8 \pi G}{c^{4}} p_{0}-\Lambda\right) r \\
\frac{1}{2} e^{-\lambda_{0}}\left(\nu_{0}^{\prime \prime}+\frac{\nu_{0}^{\prime 2}}{2}+\frac{\nu_{0}^{\prime}-\lambda_{0}^{\prime}}{r}-\frac{\nu_{0}^{\prime} \lambda_{0}^{\prime}}{2}\right)=\frac{8 \pi G}{c^{4}} p_{0}-\Lambda,
\end{gathered}
$$

where the index 0 refers to the unperturbed metric and physical quantities.

\section{APPENDIX B: PULSATION EQUATION}

In this Appendix we present the pulsation equation for a homogeneous sphere and for the trial function $\zeta=\eta^{3}$. By introducing the new variable $\theta=\arcsin (\sqrt{2 / 3+l} \eta)$, denoting $\theta_{S}=\arcsin \left(\sqrt{2 / 3+l} \eta_{S}\right)$ and taking into account that $\alpha=\cos \theta_{S} /(1 / 3-l)$, in the limit $\omega \rightarrow 0$ we obtain the following equation for $\theta_{S}$ :

$$
360\left[22-34 \gamma+9 l\left(1+14 \gamma-9 l \gamma+3(2+\gamma) l^{2}\right)\right] \theta_{S} \cos \left(\theta_{S}\right)+
$$




$$
\begin{gathered}
1080[2+3 l+3 \gamma(3 l-1)] \theta_{S} \cos \left(3 \theta_{S}\right)- \\
20\left[134-63 l+594 l^{3}+27(3 l-1)\left(7-8 l+6 l^{2}\right) \gamma\right] \sin \left(\theta_{S}\right)+ \\
5\left[-440+717 \gamma+9 l\left(-22\left(2+3 l^{2}\right)+9(-31+l(13+l) \gamma)\right)\right] \times \\
\sin \left(3 \theta_{S}\right)- \\
{\left[-10(3 l-4)(2+3 l)^{2}+27(3 l-1)(7+(8+3 l) l) \gamma\right] \times} \\
\sin \left(5 \theta_{S}\right)=0 .
\end{gathered}
$$

[1] F. E. Schunck and E. W. Mielke, Phys. Rev. D50, 4794 (1994)

[2] A. Einstein, Sitzungber. Preuss. Akad. Wiss. Phys.-Math. Kl. 1917, 142 (1917).

[3] H. Bondi and T. Gold, Mon. Not. R. Astr. Soc. 108, 252 (1948).

[4] F. Hoyle and J. V. Narlikar, Proc. Roy. Soc. A270, 334 (1962).

[5] A. H. Guth, Phys. Rev. D23, 347 (1981).

[6] A. Linde, Particle Physics and Inflationary Cosmology, Harwood Academic Publishers, (1990).

[7] Ya. B. Zeldovich and I. D. Novikov, Relativistic Astrophysics, Univ. of Chicago Press, Chicago, Ill (1971).

[8] J. Dreitlein, Phys. Rev. Lett. 33, 1243 (1974).

[9] S. Adler, Rev. Mod. Phys. 54, 729 (1982).

[10] M. Ozer and M. O. Taha, Phys. Lett. B171, 363 (1986).

[11] T. Padmanabhan, Phys. Rept. 380, 235 (2003).

[12] K. Freese, F. C. Adams, J. A. Frieman and E. Mottola, Nucl. Phys. B287, 797 (1987); B. Ratra and P. J. E. Peebles, Phys. Rev. D37, 3406 (1988); J. Lopez and D. V. Nanopoulos, Mod. Phys. Lett. A11,1 (1996); R. Brandenberger and A. Mazumdar, JCAP 0408, 015 (2004).

[13] M. Gasperini, Class. Quantum. Grav. 5, 521 (1988).

[14] A. G. Riess et al., Astron. J. 116, 1009 (1998).

[15] S. Perlmuter et al., Nature 391, 51 (1998).

[16] S. Perlmuter et al., Astrophys. J. 517, 531 (1999).

[17] J. P. Ostriker and P. J. Steinhardt, Nature 377, 600 (1995).

[18] A. G. Riess, et al., Astrophys. J. 607, 665 (2004). 
[19] P. J. E. Peebles and B. Ratra, Rev. Mod. Phys. 75, 559 (2003).

[20] F. D. Ryan, Phys. Rev. D55, 6081 (1997).

[21] E. W. Mielke and F. E. Schunck, Nucl. Phys. B564, 185 (2000).

[22] F. E. Schunk and E. W. Mielke, Class. Quantum. Grav. 20, R301 (2003).

[23] E. Seidel and W. M. Suen, Phys. Rev. D42, 384 (1990).

[24] F. Kusmartsev, E. W. Mielke and F. E. Schunck, Phys. Rev. D43, 3895 (1991).

[25] J. F. Cardona and J. M. Tejeiro, Astrophys. J. 493, 52 (1998).

[26] M. K. Mak, P. N. Dobson, Jr. and T. Harko, Mod. Phys. Lett. A15, 2153 (2000).

[27] C. G. Böhmer, Gen. Rel. Grav. 36, 1039 (2004).

[28] Z. Stuchlík, Acta Physica Slovaca 50, 219 (2000).

[29] C. G. Böhmer, Ukr. J. Phys. 50, 1219 (2005).

[30] A. Balaguera-Antolínez, C. G. Böhmer and M. Nowakowski, Int. J. Mod. Phys. D14, 1507 (2005).

[31] S. Chandrasekhar, Astrophys. J. 140, 417 (1964).

[32] M. Gleiser and R. Watkins, Nucl. Phys. B319, 733 (1989).

[33] D. Astefanesei and E. Radu, Nucl. Phys. B665, 594 (2003).

[34] R. C. Tolman, Relativity, Thermodynamics and Cosmology, Oxford, Clarendon Press (1934);

L. D. Landau and E. M. Lifshitz, The classical theory of fields, Oxford, Pergamon Press, (1975).

[35] D. Gondek, P. Haensel and J. L. Zdunik, Astron. Astrophys. 325, 217 (1997).

[36] T. Harko and M. K. Mak, Journal of Mathematical Physics 41, 4752 (2000).

[37] A. D. Rendall and B. G. Schmidt, Class. Quantum. Grav. 8, 985 (1991).

[38] J. Bardeen, K. S. Thorne and D. W. Meltzer, Astrophys. J. 145, 505 (1966).

[39] P. J. E. Peebles, Astrophys. J. 284, 439(1984).

[40] G. L. Comer and H. Shinkai, Class. Quantum Gravity 15, 669 (1998).

[41] I. Waga, Astrophys. J. 414, 436 (1993). 